\title{
Téoros
}

Revue de recherche en tourisme

\section{Le tourisme cynégétique et halieutique face au défi de la durabilité}

\section{Manu Tranquard}

Volume 32, numéro 1, 2013

Tourisme cynégétique et halieutique

URI : https://id.erudit.org/iderudit/1036648ar

DOI : https://doi.org/10.7202/1036648ar

Aller au sommaire du numéro

Éditeur(s)

Université du Québec à Montréal

ISSN

0712-8657 (imprimé)

1923-2705 (numérique)

Découvrir la revue

Citer ce document

Tranquard, M. (2013). Le tourisme cynégétique et halieutique face au défi de la durabilité. Téoros, 32(1), 3-6. https://doi.org/10.7202/1036648ar d'utilisation que vous pouvez consulter en ligne.

https://apropos.erudit.org/fr/usagers/politique-dutilisation/ 


\title{
Le tourisme cynégétique et halieutique face au défi de la durabilité
}

\author{
Manu TRANQUARD, Ph.D., LL.M. \\ Professeur \\ Université du Québec à Chicoutimi \\ Laboratoire d'Expertise et de Recherche en Plein Air (LERPA) \\ Manu_Tranquard@uqac.ca
}

Le tourisme cynégétique et halieutique est une activité de loisir permettant aux participants de procéder à diverses formes de prélèvement faunique, en milieu naturel essentiellement. À la différence des activités d'observation faunique strictes, le tourisme de chasse et pêche vise directement le prélèvement, lequel est le principal motif des séjours. Au-delà de ce dénominateur commun, les tourismes de chasse et de pêche recoupent diverses réalités, notamment au Québec. Cette forme de tourisme est par ailleurs en proie à une profonde transformation : offre, demande, structure et organisation professionnelle, nouvelle géographie mondiale, évolution des perceptions et des mentalités, etc. Le tourisme de chasse et pêche fait également naître des questions essentielles quant à sa capacité de développement durable, notamment en ce qui concerne la gestion des populations fauniques et la préservation de leurs habitats. Enfin, le tourisme de chasse et pêche est au cœur de débats concernant la fonction et l'usage des territoires, leur partage et leur valorisation, ou encore leur charge identitaire. Pratique traditionnelle dans certaines parties du globe, ou phénomène émergent perçu comme vecteur potentiel de développement local pour d'autres territoires, le tourisme cynégétique et halieutique cristallise parfois des tensions sociales. L'objectif du présent dossier est d'aborder ces questions en apportant un éclairage sur certaines réalités géographiques et sociales, sur des pratiques professionnelles, et une mise en perspective avec l'activité touristique au sens large.

\section{Le tourisme cynégétique et halieutique : caractéristiques définitionnelles}

Le tourisme de chasse et pêche est essentiellement un tourisme de nature (nature based tourism, en anglais), c'est-àdire une forme de tourisme à laquelle peuvent être associés les produits et activités touristiques utilisant l'environnement naturel pour se réaliser, se concrétiser (Couture, 2002 : 9). Le tourisme de nature correspond à « une forme de tourisme qui dépend principalement d'un milieu naturel en tant que principal attrait ou site pour prendre forme» (Weaver, 2001 : 659). Les activités touristiques sont axées sur l'observation ou l'appréciation de la nature à des fins de découverte ou de pratique d'activités de plein air. Le tourisme de nature induit par ailleurs une démarche participative des clientèles, une implication sensible et émotionnelle, une participation plutôt qu'une consommation. Il s'agit pour les clients de vivre une expérience, d'être acteurs de leur découverte (Macouin et Pierre, 2003 : 30; Breton, 2006). Cette pratique touristique favorise donc l'immersion dans le milieu naturel d'accueil et lui confère un rôle plus important que pour d'autres catégories de tourisme. Au sens de ces définitions, le tourisme de chasse et pêche est un tourisme de nature, même si certaines pratiques, par exemple la pêche blanche offerte dans des pourvoiries urbaines sur des sites constitués de villages sur glace, montrent leurs limites de ce qui peut être considéré comme relevant de la nature. Il reste que les ressources naturelles (environnement biophysique incluant les ressources fauniques) sont à la base de l'offre, car d'elles dépend l'initialisation du circuit économique.

Parmi ses autres caractéristiques définitionnelles, l'objectif du tourisme de chasse et pêche est de faire vivre une expérience de prélèvement faunique. Cette expérience peut varier selon le type d'activité pratiqué. Pour le tourisme de pêche, par exemple, le prélèvement n'inclut pas systématiquement, à la différence des activités cynégétiques, la mise à mort des espèces recherchées. En fonction des territoires sur lesquels se déroule l'activité, des réglementations en vigueur, du statut des espèces, etc., le tourisme halieutique peut donner lieu à la remise à l'eau des captures (pratique de catch and release). Les activités touristiques de chasse et pêche peuvent par ailleurs être guidées ou autoguidées, selon que les participants sont accompagnés ou autonomes durant leur séjour. Là encore, l'option peut relever d'un choix personnel, ou le recours à un guide peut être imposé par une 
réglementation ou un contexte spécifique. Ainsi au Québec et en vertu de la Loi sur la conservation et la mise en valeur de la faune (Québec, 2013), les non-résidents doivent utiliser les services d'un pourvoyeur pour pratiquer la pêche sportive et chasser toute espèce de gibier au nord du $52^{\mathrm{e}}$ parallèle. Autre constat, le profil des clientèles du tourisme de chasse et celui du tourisme de pêche se distinguent traditionnellement l'un de l'autre : plus masculin et international pour le premier, il est généralement plus familial et grand public pour le second. Un dernier constat générique d'importance est que le tourisme de chasse et pêche est un tourisme profondément identitaire et patrimonial. Il fait souvent appel à des pratiques locales et traditionnelles, aussi diverses que les populations fauniques peuvent l'être. Il s'inscrit ainsi dans une histoire territoriale. L'instauration puis le démantèlement des clubs privés de chasse et pêche illustre cet ancrage dans l'histoire du Québec (Gagnon, 2009).

Ces précisions apportées, une question demeure : le tourisme cynégétique et halieutique est-il aujourd'hui une réalité économique et commerciale courante ou son existence se limite-t-elle à des pratiques confidentielles, très géographiquement circonscrites? La question se pose en dépit des principes mentionnés plus haut, car force est de constater que la pratique de la chasse et de la pêche dans un cadre touristique ne va à priori pas de soi. Longtemps activités de subsistance, la chasse et la pêche se sont tardivement muées en activités de loisirs récréatifs. Toutefois, de là à servir de motifs pour des expériences touristiques, le pas n'est pas nécessairement franchi à l'échelle mondiale. Une illustration de cela est notamment les amalgames souvent faits dans la terminologie entourant ces activités. Il est ainsi courant de confondre la pêche sportive avec le tourisme halieutique. La pêche sportive fait référence à une activité de loisir, pratiquée de manière autonome, dans un but désintéressé, en opposition à la pêche commerciale. Le tourisme halieutique peut reposer sur le prélèvement des mêmes espèces animales, mais dans le cadre d'une activité touristique, souvent guidée, intégrant déplacement et séjour sur un site d'intérêt biogéophysique mais aussi patrimonial, historique, etc. Dans un contexte où l'industrie touristique est de plus en plus portée à proposer des expériences globales aux visiteurs, la distinction entre ces deux pratiques est importante. Dans les faits, il existe bien un tourisme de chasse et pêche au Québec ou encore sur le continent africain (les fameux safaris). Dans d'autres territoires, la chasse et la pêche reposent presque exclusivement sur des loisirs récréatifs individuels (la France en est un exemple), la dimension touristique étant pratiquement absente.

Au-delà de la question des contours définitionnels de cette forme de tourisme, plusieurs défis trouvent à se poser sur les territoires où elle s'implante, concernant sa pérennisation, voire son développement durable. À ce titre, une étude relativement récente sur le tourisme cynégétique et halieutique au Québec (KPMG, 2010) relève un certain nombre de données clés concernant les enjeux et tendances de cette industrie. Ces données peuvent nourrir la réflexion scientifique, notamment parce qu'elles recoupent des constats identiques faits à l'international.

\section{Enjeux et défis}

Le tourisme cynégétique et halieutique est actuellement confronté à l'évolution des mentalités et des pratiques. Il est globalement tributaire de la baisse de popularité des activités de chasse et pêche qui résulte en partie d'un problème d'image auprès d'une portion de la population alors même qu'on assiste à la croissance du tourisme de nature, lequel représente aujourd'hui près du quart de la demande touristique mondiale (KPMG, 2010 : 3). Une des raisons de cette situation tiendrait au fait que les touristes sont de plus en plus conscients des enjeux liés à la protection de l'environnement et notamment à la préservation de la ressource faunique. Cependant, un déficit d'informations concernant la compatibilité de cette forme de tourisme avec les préoccupations environnementales pourrait jouer en la défaveur de son développement. Cette mentalité est présente alors même que les méthodes de gestion des populations fauniques n'ont jamais été aussi éprouvées scientifiquement qu'aujourd'hui.

Au niveau de l'offre, alors que dans le passé le voyageur international était attiré par une forte image de marque de la destination Québec (destination nature, Grand-Nord, présence d'une grande faune sauvage, possibilité de trophées), l'abondance actuelle de produits pourvoiries - chasse et pêche non différenciées - exacerbe le niveau de compétitivité. Ceci va à l'encontre de la recherche d'authenticité en ce qui a trait aux produits touristiques, autre tendance majeure de l'industrie. Dans un contexte où la clientèle de chasseurs et de pêcheurs semble ne pas se renouveler suffisamment, l'importance de l'offre conduit parfois même au développement de pratiques non souhaitables (ex. : économie au noir, chasse garantie). Le tourisme cynégétique et halieutique subit par ailleurs les conséquences de l'accroissement de la pratique autonome dans les disciplines «sportives» de plein air qui conduit de nombreux adeptes à ne plus recourir à des services touristiques professionnels. Il doit enfin cohabiter avec un certain nombre d'autres formes de tourisme de nature au rang desquels se trouvent le tourisme écologique et le tourisme d'observation faunique.

À contrario, de nombreux professionnels du tourisme de prélèvement faunique vivent actuellement une «détraditionnalisation» de leurs métiers et produits. On assiste ainsi, au Québec notamment, à l'évolution de certaines pourvoiries vers le "multiactivités», ou encore à la tentative d'établissement de nouveaux standards dans l'exercice de la profession de guide de chasse et pêche. Ailleurs dans le monde, de nouvelles avenues sont entrevues pour contribuer au renouvellement et à l'enrichissement des produits, tel le pescatourisme comme complément d'activité pour les marins pêcheurs. Des pistes sont donc envisagées pour contrer la stagnation de la demande, problématique d'autant plus importante que le tourisme cynégétique et halieutique est parfois une composante majeure de la stratégie de développement socio-économique de certaines régions ressources. Le Grand-Nord québécois en est un exemple : le renouvellement de la clientèle tend à y être être favorisé par un produit pourvoirie tablant sur la villégiature ou par l'augmentation des marchés spécialisés ou de niche. Ailleurs, les entreprises étrangères performantes privilégient 
des expériences touristiques fortement enrichies par la qualité et l'unicité du produit. Ainsi les régions internationales émergentes font des gains en misant sur des expériences uniques et des voyages conçus selon les goûts et les attentes de la clientèle, et en investissant dans la formation et la certification des guides.

Plus globalement, le principal défi du tourisme cynégétique et halieutique semble reposer sur sa capacité à intégrer les principes d'un développement durable. Au cours des dernières années, le tourisme durable a en effet fait l'objet d'une large promotion d'esprit normatif par des institutions internationales (OMT, 1999; AFIT, 2001). Des interrogations demeurent cependant quant à ses bases théoriques (Lauriol, 2004) ou quant à sa capacité opérationnelle (Céron et Dubois, 2002; Ryan, 2002; Marsat, 2008). Selon l'Agence française d'ingénierie touristique (AFIT, 2001), le tourisme durable désigne toute forme de développement, d'aménagement ou activité touristique qui respecte et préserve à long terme les ressources naturelles, culturelles et sociales, et contribue de manière positive et équitable au développement économique. Le tourisme cynégétique et halieutique peut-il prétendre à cette définition? Il serait alors appelé à s'inscrire rapidement dans une logique de durabilité si ses acteurs souhaitent garantir sa soutenabilité économique, écologique et sociale. Cependant, a-t-il le potentiel et les moyens de contribuer efficacement au développement durable du territoire qui porte ses activités? Le secteur a-t-il la capacité d'intégrer respect de l'environnement, viabilité économique et implication effective des communautés locales dans une perspective de transformation à long terme?

Un enjeu crucial est notamment celui de la conservation et de la gestion durable des populations animales faisant l'objet de prélèvements et notamment la raréfaction de certaines espèces emblématiques (ours polaires ou, plus récemment, caribous). En matière de chasse et pêche, les ressources naturelles fondent l'offre touristique. Les critères qui permettent de les évaluer sont leur qualité, leur cohérence et leur accessibilité. La dégradation de leur qualité, la limitation de leur accessibilité impactent donc directement l'offre et le développement économique du secteur. Plus ces ressources sont sollicitées, plus leur valeur s'amenuise, tout comme se restreint l'expérience des visiteurs et ainsi la valeur de l'offre. L'altération de certains écosystèmes, notamment des cycles biologiques des populations fauniques en raison des contacts répétés avec des visiteurs, conduit à réduire inéluctablement la capacité des générations futures à bénéficier de leur observation, voire de leur prélèvement dans un cadre d'expérience touristique.

Un autre enjeu concerne la question du partage des territoires et des pratiques. Tourisme de chasse et pêche et tourisme de nature, incluant l'observation faunique, sont en effet souvent sources de heurts et de difficultés en matière de gestion de la sécurité et de cohabitation des activités. L'acceptabilité sociale du tourisme cynégétique et halieutique et la gestion des conflits d'usage sont des questions actuelles. La capacité de contribution du tourisme de chasse et pêche au développement durable repose enfin sur l'activation et la pérennisation d'une économie locale viable et en croissance.
Dans le droit fil de ces questions centrales, Christophe BATICLE, socioanthropologue à l'Université de Picardie Jules Verne, interroge dans ce numéro de Téoros la manière dont le loisir chasse se conçoit en France en s'intéressant plus particulièrement aux raisons sociales, anthropologiques et culturelles qui font que l'activité résiste à une mise en tourisme. L'enjeu essentiel est l'ouverture des territoires fauniques à l'usage marchand alors même que ceux-ci sont très largement investis d'une signification identitaire qui semble interdire leur exploitation. Outre la symbolique du territoire qui serait donc un frein à son exploitation touristique, la régulation juridique du droit de chasse le serait également.

À partir d'une étude de cas portant sur la réserve faunique de Matane (Québec), Élaine CHANTELOUP, de l'Université de Savoie - Laboratoire Edytem — et de l'Université de Montréal, aborde également la question de la mise en tourisme de la chasse et des obstacles liés à la perte de maîtrise des territoires. La question est traitée par le biais d'une analyse comparative des activités touristiques consommatrices et non consommatrices de la faune sauvage. La mise en place de projets de territoire ou de systèmes touristiques localisés pourrait favoriser l'acceptation sociale de la mise en partage du territoire.

L'article de Vincent LECLERC et Marc-Antoine VACHON, de l'Université du Québec à Montréal, étend le champ des défis du tourisme cynégétique en abordant les questions des conflits entre usagers, l'image négative de la chasse, les difficultés de promotion de la chasse et le manque de relève. Plus précisément, cette contribution traite de la méconnaissance du marché comme point d'achoppement du développement de l'activité. En discussion, des pistes de solutions et de recherche sont proposées.

$\mathrm{Au}$ chapitre des options de développement durable du tourisme cynégétique et halieutique, Erwann CHARLES et Thierry SAUVIN, de l'Université de Bretagne Occidentale, traitent de la question de la labélisation comme outil de valorisation d'une ressource territoriale en contexte touristique. En analysant le cas du label "Accueil Pêche en Finistère", les auteurs font la démonstration qu'une stratégie visant à construire un véritable territoire de projet autour d'un tourisme environnemental ou écologique est possible s'agissant d'un tourisme de prélèvement faunique. La ressource halieutique est ici intégrée dans le cadre d'un programme qualité visant son exploitation touristique autour de valeurs communes partagées, traduites tant par la «gouvernance» mise en place que par les «atouts» mis en avant au niveau local.

Frédérick GUYON, de l'Université de Franche-Comté, propose pour sa part une étude de modes de construction des territoires touristiques permettant à une offre de tourisme de prélèvement faunique d'exister. L'analyse porte essentiellement sur les discours et les offres des promoteurs du tourisme de chasse et du tourisme de pêche. L'approche est celle du développement local. La «culture locale» et le sens géoanthropologique des territoires (Gagnon, 2009) influeraient sur la gestion et le développement des activités touristiques. En soulignant les spécificités du cas français, cette contribution alimente la réflexion sur la gouvernance du tourisme cynégétique et halieutique. 
Enfin, Dominique VOLLET, du Cemagref UMR Métafort, livre une étude de l'impact économique de la chasse sur l'emploi local en territoire français. À partir d'une analyse économique de type méthodologique, l'auteur s'intéresse aux possibilités de contribution du tourisme au développement territorial durable. L'outil méthodologique peut alors devenir un support à la mise en place de stratégies locales efficientes.

\section{Références}

AFIT - Agence française de l'ingénierie touristique (2001) Piloter le tou-

risme durable dans les territoires et les entreprises, Paris : Atout France. $127 \mathrm{p}$.

BRETON, Jean-Marie (sous la direction de) (2006) Développement viable et valorisation environnementale, Pointe-à-Pitre : Éditions Karthala. $486 \mathrm{p}$.

CÉRON, Jean-Paul et Ghislain DUBOIS (2002) Le tourisme durable dans les destinations. Guide d'utilisation, Limoges : Presses de l'Université de Limoges, CRIDEAU-CNRS. $170 \mathrm{p}$.

COUTURE, Maurice (2002) «L'écotourisme - un concept en constante évolution", Téoros, vol. 21, n 3 , automne, p. 5-13.

GAGNON, Serge (2009) «L'appropriation ludique de la forêt au Québec : d'une gestion privée de "club" à une intervention publique de protection (1885-1935) », Études caribéennes, <http://etudescaribeennes. revues.org/3609>, consulté le 14 août 2013.
KPMG Services Conseils (2010) Diagnostic - Pourvoiries — chasse et pêche, Québec: Gouvernement du Québec. 40 p., <http://www.tourisme.gouv.qc.ca/publications/media/document/etudes-statistiques/ diagnostic-chasse-peche.pdf>, consulté le 11 mars 2012.

LAURIOL, Jacques (2004) «Le développement durable à la recherche d'un corps de doctrine", Revue française de gestion, vol. 30, nº 152, p. 137-150.

MACOUIN, Dominique et Isabelle PIERRE (2003) Le tourisme de nature, AFIT, Paris : Éditions Atout France. 43 p.

MARSAT, Jean-Bernard (2008) «Valorisation et gestion des aménités naturelles : Comment construire des stratégies d'alliance entre acteurs d'environnement et acteurs du tourisme? ». Thèse de doctorat, Paris : AgroParisTech. 668 p.

OMT — Organisation mondiale du tourisme (1999) Guide à l'intention des autorités locales : développement durable du tourisme, Paris : OMT. $223 \mathrm{p}$.

Québec (2013) «LRQ, c C-61.1», Loi sur la conservation et la mise en valeur de la faune, Québec : Éditeur officiel du Québec, <http://www.canlii. org/fr/qc/legis/lois/lrq-c-c-61.1/derniere/lrq-c-c-61.1.html $>$. À jour au 11 septembre 2009.

RYAN, Chris (2002) «Equity, management, power sharing and sustainability — issues of the "new tourism" ", Tourism Management, vol. 23, $\mathrm{n}^{\circ}$ 1, p. 17-26.

WEAVER, David Bruce (2001) The Encyclopaedia of Ecotourism, Wallingford : Cabi Publising. 668 p.

\section{Appel à textes}

En dehors des dossiers thématiques, Téoros ouvre ses pages aux propositions spontanées. La rédaction invite les chercheur(e)s qui œuvrent dans le domaine du tourisme ou qui s'intéressent au tourisme sous tous ses aspects à soumettre des articles de nature analytique à la revue. On peut soumettre un article en l'envoyant à :

\section{teoros@uqam.ca}

Les textes soumis doivent apporter une contribution scientifique originale, que ce soit par le biais d'information factuelle jusqu'alors inconnue ou par une nouvelle interprétation d'un thème particulier. Téoros vise avant tout le transfert de connaissances; son objectif est donc de promouvoir une meilleure compréhension des phénomènes liés au tourisme.

Les auteurs doivent faire parvenir un manuscrit présenté selon les règles de la revue, disponibles au teoros.revues.org. Un article compte environ 7000 mots et n'excède pas 7500 mots, avec trois ou quatre illustrations en 300 ppp. Les articles peuvent être soumis en anglais ou en français et doivent être accompagnés d'un résumé de 200 mots et de cinq mots-clés.

La publication des articles se fait sous réserve d'une évaluation. Tous les manuscrits seront évalués anonymement par des pairs, qui pourront faire des suggestions ou demander des modifications. La rédaction transmettra l'avis des évaluateurs aux auteurs et s'assurera que les modifications demandées seront apportées.

Au plaisir de vous lire dans nos pages.

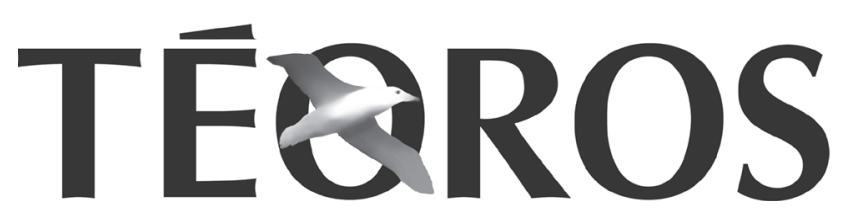

